\section{REVISTA BRASILEIRA DE QUALIDADE DE VIDA}

\title{
Cálculo dos escores e estatística descritiva do WHOQOL-8 por meio do Microsoft Excel
}

\section{Calculation of scores and descriptive statistics of WHOQOL-8 through Microsoft Excel}

\author{
Bruno Pedroso \\ Universidade Estadual de Ponta Grossa - UEPG - Ponta Grossa - Paraná - Brasil \\ prof.brunopedroso@gmail.com \\ Bruna Maria Palotino Ferreira \\ Universidade Estadual de Ponta Grossa - UEPG - Ponta Grossa - Paraná - Brasil \\ bruna_mpalotinof@hotmail.com
}

Com o objetivo de construir um instrumento de avaliação da qualidade de vida para o Projeto EUROHIS, que intentava a elaboração de questionários e entrevistas para avaliação de indicadores da área da saúde no continente europeu, fora convencionado que se tentaria adaptar um instrumento já existente para uma versão com um pequeno número de questões para pesquisas em nível nacional, tais quais os censos demográficos (POWER, 2003).

O projeto do novo instrumento foi batizado de EUROHIS-QOL (POWER, 2003; SCHMIDT; MÜHLAN; POWER, 2006). Posteriormente, também recebeu a denominação de WHOQOL-8 (AUSTRALIAN WHOQOL FIELD CENTRE, 2006).

Para a construção do WHOQOL-8, foram analisados os dados do teste piloto do WHOQOL - utilizado para a construção do WHOQOL-100 -, do teste de campo do WHOQOL-100 e do teste de campo do WHOQOL-bref. Fora decidido que os itens a serem selecionados deveriam estar presentes tanto no WHOQOL-100 quanto no WHOQOL-bref (POWER, 2003).

As questões, pertencentes ao WHOQOL-bref, selecionadas para a composição do WHOQOL-8 foram:

Quadro 1 - Questões do WHOQOL-8

\begin{tabular}{|c|l|}
\hline NUMERAÇÃO & \multicolumn{1}{c|}{ QUESTÕES } \\
\hline F1 & Como você avaliaria sua qualidade de vida? \\
\hline F2 & Quão satisfeito(a) você está com a sua saúde? \\
\hline F10 & Você tem energia suficiente para seu dia-a-dia? \\
\hline F17 & $\begin{array}{l}\text { Quão satisfeito(a) você está com sua capacidade de desempenhar as } \\
\text { atividades do seu dia-a-dia? }\end{array}$ \\
\hline F19 & Quão satisfeito(a) você está consigo mesmo? \\
\hline F20 & $\begin{array}{l}\text { Quão satisfeito(a) você está com suas relações pessoais (amigos, } \\
\text { parentes, conhecidos, colegas)? }\end{array}$ \\
\hline F12 & Você tem dinheiro suficiente para satisfazer suas necessidades? \\
\hline F23 & Quão satisfeito(a) você está com as condições do local onde mora? \\
\hline \multicolumn{2}{|l}{ Fonte: Adaptado de Power (2003) e Schmidt, Mühlan e Power (2006). }
\end{tabular}


O WHOQOL-8 se demonstra como uma alternativa viável para utilização em grandes populações ou em situações em que se demande de pouco tempo para o preenchimento do questionário. Ressalta-se, também, a possibilidade de sua aplicação em conjunto com outras pesquisas, em que seja pertinente levantar dados relacionados à qualidade de vida. Também ficou evidenciada a viabilidade de sua aplicação por meio de entrevistas por telefone.

No entanto, a obrigatoriedade de utilização do software Statistical Package for Social Sciences (SPSS), imposta pelo Grupo WHOQOL, constitui um fator limitante em sua utilização, ao passo que este requer conhecimentos específicos e é de custo elevado.

Objetivando suprimir essa limitação, a partir da sintaxe para o cálculo do escore do WHOQOL-8 proposta pelos seus desenvolvedores, construiu-se no mesmo molde da ferramenta desenvolvida por Pedroso et al. (2010) uma ferramenta para o cálculo dos escores e da estatística descritiva do WHOQOL-8.

A ferramenta proposta no presente trabalho realiza automaticamente o cálculo dos escores e da estatística descritiva do WHOQOL-8. Ao pesquisador cabe apenas preencher as respostas obtidas nos questionários aplicados. Suas características são:

a) As células cujas respostas estão corretas (respondidas com valores situados no intervalo compreendido entre 1 e 5) são representadas através da cor de preenchimento verde;

b) Caso algum respondente tenha deixado de responder alguma questão, o pesquisador será instruído a excluir tal respondente da amostra. O número de questões não respondidas é informado ao pesquisador e estas estarão em destaque (cor do preenchimento branco);

c) Caso alguma resposta tenha sido preenchida com algum valor que não conste entre o intervalo de 1 a 5, o pesquisador será instruído a excluir tal respondente da amostra. $\mathrm{O}$ número de respostas inválidas será notificado ao pesquisador e estas estarão em destaque (cor do preenchimento vermelho);

d) O escore global do WHOQOL-8 de cada respondente consiste no cálculo da soma dos escores das oito questões do instrumento seguido da subtração de oito unidades, perfazendo um resultado entre 0 e 32 ;

e) A estatística descritiva das questões e o escore global do WHOQOL-8 correspondente à média, ao desvio padrão, ao valor máximo, ao valor mínimo, ao coeficiente de variação e à amplitude é calculada;

f) O pesquisador poderá copiar os escores individuais de cada respondente, os resultados de estatística descritiva e os gráficos, mas não pode modificar os resultados. $\mathrm{O}$ único local que lhe é permitido a inserção e edição de valores é a área de tabulação das respostas.

Para testar a fidedignidade da ferramenta proposta, fora realizada uma aplicação do WHOQOL-8 em 56 indivíduos. Os cálculos dos resultados foram realizados por meio da ferramenta construída no presente estudo e, também, por meio da sintaxe SPSS proposta pelos desenvolvedores do WHOQOL-8. Os resultados retornados pela ferramenta foram comparados com os resultados retornados pelo SPSS, tendo havido 100\% de coincidência entre ambos (FERREIRA, 2015).

O download da referida ferramenta pode ser realizado do sítio eletrônico http://www.brunopedroso.com.br/whoqol-8.html.

Conclui-se que a partir do presente estudo foi possível a disponibilização de uma ferramenta alternativa, fidedigna à sintaxe original, que facilita o acesso aos resultados de aplicações do WHOQOL-8, possibilitando a obtenção dos resultados deste por meio de uma interface simples e sem a necessidade de aquisição de softwares para fins específicos e que requerem conhecimentos avançados de utilização. 


\section{Referências}

AUSTRALIAN WHOQOL FIELD CENTRE. Use the World Health Organization Quality of Life (WHOQOL) Instruments. Centre for Participant Report of University of Melbourne,

Melbourne, 2006. Department of Psychiatry. Disponível em:

<http://www.psychiatry.unimelb.edu.au/centres-units/cpro/whoqol/use-whoqol.html >. Acesso em 23 abr. 2012.

FERREIRA, B. M. P. Cálculo dos escores e estatística descritiva do instrumento WHOQOL-8 utilizando o Microsoft Excel. 2015. 16 f. Monografia (Graduação em Bacharelado em Educação Física) - Departamento de Educação Física, Universidade Estadual de Ponta Grossa, Ponta Grossa, 2015.

PEDROSO, B. et al. Cálculo dos escores e estatística descritiva do WHOQOL-bref através do Microsoft Excel. Revista Brasileira de Qualidade de Vida, v. 2, n. 1, p. 31-36, jan./jun. 2010. crossef

POWER, M. J. Development of a common instrument for quality of life. In: NOSIKOV, A.; GUDEX, A. (Org.). EUROHIS: developing common instruments for health surveys. 1. ed. Amsterdam: IOS Press, 2003.

SCHMIDT, S.; MÜHLAN, H.; POWER, M. J. The EUROHIS-QOL 8-item index: psychometric results of a cross-cultural field study. European Journal of Public Health, v. 16, n. 4, p. 420-428, Aug. 2006. Crossef 